\title{
Faire Entscheidungsprozesse und Gerechtigkeit in der
} Gesundheitsversorgung: das Beispiel von Norman Daniels' Accountablity for Reasonableness ${ }^{1}$

\section{ANNETTE RID}

Dr. med. Annette Rid ist Oberassistentin am Institut für Biomedizinische Ethik der Universität Zürich und Assistentsärztin für Klinische Ethik am UniversitätsSpital Zürich.

Die gerechte Verteilung von knappen Mitteln für die Gesundheitsversorgung stellt nicht nur eine theoretische Herausforderung dar. In der Praxis müssen Allokationsentscheidungen auch dann getroffen werden, wenn sich vernünftige Leute darüber uneinig sind, welche Entscheidungen ethisch vertretbar sind. Faire Entscheidungsverfahren spielen in dieser Situation eine besondere Rolle. Der vorliegende Beitrag diskutiert den wahrscheinlich einflussreichsten Ansatz für faire Entscheidungsverfahren im Gesundheitswesen, Norman Daniels' und James Sabin's accountability for reasonableness ('Verantwortung für Vernünftigkeitı).

\section{Einleitung}

Die gerechte Verteilung von knappen Mitteln für die Gesundheitsversorgung stellt nicht nur eine theoretische Herausforderung dar. In der Praxis müssen Allokationsentscheidungen auch dann getroffen werden, wenn sich vernünftige Leute darüber uneinig sind, welche Entscheidungen ethisch vertretbar sind. Faire Entscheidungsverfahren spielen in dieser Situation eine besondere Rolle. Wenn es keine allgemein anerkannten Gerechtigkeitskriterien für die Verteilung von knappen Mitteln gibt, aber dennoch Entscheidungen getroffen werden müssen, die einzelne Patienten oder ganze Patientengruppen benachteiligen können, dann sollten diese Entscheidungen auf faire Weise getroffen werden.
Ausgehend von dieser grundlegenden Einsicht haben zwei amerikanische Autoren - der Philosoph Norman Daniels und der Mediziner James Sabin - Ende der 1990er Jahre ein Modell für faire Entscheidungsverfahren im Gesundheitswesen entwickelt [3].

Das Modell der >Verantwortung für Vernünftigkeit< (accountability for reasonableness) hat die Praxis der Prioritätensetzung im Gesundheitswesen ebenso beeinflusst wie theoretische De-

\footnotetext{
1 Der vorliegende Beitrag beruht auf einem Artikel, der durch den Schweizerischen Nationalfonds unterstützt und im Januar 2009 im Journal of Medical Ethics veröffentlicht wurde [1]. Eine vollständige deutsche Übersetzung ist 2010 in einem Sammelband zu Public Health Ethik erschienen, der von Daniel Strech und Georg Marckmann herausgegeben wurde [2].
} 
batten über Gesundheitsgerechtigkeit. Zahlreiche Institutionen im Gesundheitsbereich arbeiten mit dem Modell der >Verantwortung für Vernünftigkeit . Die Weltgesundheitsorganisation hat im Rahmen der $>3$ by 5 - Initiative, die in Entwicklungsländern die Versorgung mit anti-retroviralen Medikamenten verbessern sollte, die Prinzipien der -Verantwortung für Vernünftigkeit befürwortet [4]. Das britische National Institute of Clinical Excellence (NICE) orientiert sich bei seinen Entscheidungen über die Effektivität und Kosten-Effektivität von medizinischen Interventionen ebenfalls an diesen Prinzipien [5]. Auch in Mexiko hat die >Verantwortung für Vernünftigkeit` Entscheidungen über die Ausweitung der Grundversorgung durch das nationale Gesundheitssystem beeinflusst [6]. Medizinische Institutionen und Abteilungen nutzen das Modell zur Evaluation und Verbesserung ihrer Priorisierungsprozesse [7-9]. Aber auch unter Akademikern ist das Modell der ,Verantwortung für Vernünftigkeit breit diskutiert worden. Eine Reihe von empirischen Studien gleichen das Modell mit den Werten und Zielen von Akteuren im Gesundheitswesen $a b$ und finden insgesamt große Übereinstimmung [10-11]. Auch aus theoretischer Perspektive ist der Ansatz in zahlreichen Arbeiten beleuchtet und hinterfragt worden, vor allem aufgrund der Unbestimmtheit der Bedingungen für ein faires Entscheidungsverfahren [12-14].

Der vorliegende Beitrag reiht sich in die letzte Gruppe von Abhandlungen zur >Verantwortung für Vernünftigkeit<. Vor einigen Jahren hat Norman Daniels ein Buch veröffentlicht, das seine Arbeiten zu fairen Entscheidungsverfahren mit seinen bis dahin weitgehend unabhängigen Überlegungen zur Gesundheitsgerechtigkeit zusammenführt [6]. Die Verbindung von Daniels' allgemeiner Theorie der Gesundheitsgerechtigkeit mit einem verfahrensorientierten Ansatz zur Lösung konkreter, gesundheitsbezogener Gerechtigkeitsprobleme erlaubt interessante Rückschlüsse auf das Modell der >Verantwortung für Vernünftigkeit . Dadurch können einige offene Fragen zu diesem einflussreichen Ansatz genauer geklärt werden. Wie lassen sich die Bedingungen der >Verantwortlichkeit für Vernünftigkeit am besten verstehen? Wie genau führt der Ansatz zu fairen Allokationsentscheidungen? Und weist das Modell der >Verantwortlichkeit für Vernünftigkeit< möglicherweise auch Lücken auf? Der vorliegende Beitrag widmet sich diesen Fragen mit dem Ziel, die Bedingungen der $>$ Verantwortlichkeit für Vernünftigkeit sowohl theoretisch als auch praktisch besser greifbar zu machen. Gerade in Deutschland, wo die Bewertung von Arzneimitteln und Diagnose- und Therapieverfahren durch das Institut für Qualität und Wirtschaftlichkeit im Gesundheitswesen (IQWIG) und Entscheidungen über den Umfang des Leistungskatologs der gesetzlichen Krankenversicherung (GKV) im Gemeinsamen Bundesausschuss (G-BA) noch weitgehend intransparent erfolgen, kann damit hoffentlich die Diskussion um faire Entscheidungsprozesse im Gesundheitswesen vorangetrieben werden.

\section{Die praktische Notwendigkeit von fairen Entscheidungsverfahren}

Um den Ansatz der >Verantwortung für Vernünftigkeit $<$ hinreichend zu verstehen, bedarf es einen kurzen Exkurses zu Norman Daniels' allgemeiner Theorie der Gesundheitsgerechtigkeit. Denn erst aus dem Hauptdefizit dieser Theorie - das die meisten anderen Theorien der Gesundheitsgerechtigkeit allerdings genauso teilen - ergibt sich die praktische Notwendigkeit von fairen Entscheidungsverfahren. Nach Daniels hat Gesundheit deswegen eine besondere moralische Relevanz, weil sie maßgeblich zu den uns offenstehenden Chancen und Möglichkeiten (opportunities) und damit zur fairen Chancengleichheit (fair equality of opportunity) innerhalb einer Gesellschaft beiträgt. Wenn wir aus Gerechtigkeitsgründen soziale Pflichten zum Schutz der Chancen und Möglichkeiten jedes Einzelnen haben, wie Daniels im Anschluss an den Philosophen John Rawls argumentiert [15], dann ist die Beförderung und Wiederherstellung von Gesundheit eine dieser Pflichten. Um faire Chancengleichheit zu gewährleisten, muss eine gerechte Gesellschaft ihren Mitgliedern diejenigen Gesundheitsleistungen zugänglich machen, die zur Erhaltung der normalen Spannbreite an Chance und Möglichkeiten (normal opportunity range) notwendig sind.
Doch obwohl das Prinzip der fairen Chancengleichheit allgemeine Orientierung in Fragen der Gesundheitsgerechtigkeit bieten kann, stellt sich für Daniels ein Problem. Das Prinzip gibt wenig praktische Handlungsanweisungen in Situationen, in denen die Mittel für Gesundheitsversorgung besonders knapp sind - und zwar auch dann, wenn zusätzlich Rawls' andere Gerechtigkeitsprinzipien berücksichtigt werden. ${ }^{2}$ Daniels betont immer wieder, dass das Prinzip der fairen Chancengleichheit ür Daniels ist das Prinzip der fairen Chancengleichheit zu allgemein zur Lösung von erteilungsproblemen im Cesundheitswesen.

$\mathrm{zu}$ allgemein und unbestimmt ist, um reale Verteilungsprobleme im Gesundheitswesen zeitnah lösen zu können [6, S. 24, 47, 103, 107]. Aus diesem Grund, so Daniels, sind sich >vernünftige Leute uneinig< (reasonable people disagree), wie Gesellschaften unter Bedingungen der Mittelknappheit den Gesundheitsbedürfnissen ihrer Mitglieder nachkommen sollen.

Zur Verdeutlichung dieser Situation sei eines von Daniels' eigenen Beispielen angeführt (in leicht abgewandelter Form), das ein in Industrieländern typisches Allokationsproblem veranschaulichen soll [6, S. 135]). Der G-BA überlegt, zur Behandlung fortgeschrittenen Brustkrebses eine neue hochdosierte

\footnotetext{
2 In aller Kürze zusammengefasst vertritt John Rawls, dass ein (idealisierter) Gesellschaftsvertrag zwischen freien und gleichen Bürgern drei allgemeine Gerechtigkeitsprinzipien beinhalten würde bzw. sollte: ein Prinzip zum Schutz fundamentaler Freiheiten (liberty principle); ein Prinizip zur Gewährleistung fairer Chancengleichheit (principle of fair equality of opportunity); und ein Prinzip, nach dem soziale und ökonomische Ungleichheiten in der Gesellschaft die Lage der Schlechtestgestellten bestmöglich verbessern müssen (difference prinple) [15]. Gesundheit und Gesunheitsversorgung sind für Ralws nicht gerechtigkeitsrelevant, da er von einer idealisierten Vertragssituation ausgeht, in der alle Mitglieder der Gesellschaft frei von Krankheiten und Behinderung sind. Daniels' Theorie der Gesundheitsgerechtigkeit ist eine Ausweitung von Rawls' Theorie der Gerechtigkeit auf den Bereich der Gesundheit und Gesundheitsversorgung.
} 
Chemotherapie mit Stammzelltherapie in den Leistungskatalog der GKV aufzunehmen. Die Daten zur klinischen Wirksamkeit dieser Behandlung zeigen, dass sie bei etwa zwei Drittel derjenigen Patientinnen, die alle anderen Behandlungsmöglichkeiten ausgeschöpft haben, zu einer fünfmonatigen Verlängerung der Lebenserwartung führen. Allerdings ist die Behandlung sehr teuer. Im G-BA besteht deutliche Uneinigkeit darüber, ob die zur Verfügung stehenden Mittel für effektivere Therapien genutzt werden sollten, oder ob der Patientengruppe der terminal Brustkrebserkrankten eine letzte Behandlungschance gewährt werden sollte.

Nach Daniels sind Situationen wie diese eher die Norm als die Ausnahme. Auch vernünftige Leute sind sich oftmals uneinig, wie in der Gesundheitsversorgung Grenzen gezogen werden sollen. Das praktische Problem für Daniels' Theorie der Gesundheitsgerechtigkeit - ebenso wie für die meisten anderen Theorien - aber ist, dass das Prinzip der fairen Chancengleichheit solche ungelösten Verteilungsprobleme nicht zu entscheiden vermag. Dafür ist es einfach zu unbestimmt. Doch in der Praxis müssen Allokationsentscheidungen getroffen werden. Daniels löst dieses Problem, indem er in Situationen vernüntiger Uneinigkeit (reasonable disagreement) den Schwerpunkt von substantieller Gerechtigkeit auf prozedurale Gerechtigkeit verlegt. Die zugrundeliegende Annahme ist, dass inhaltlich ausgerichtete Gerechtigkeitskriterien wie zum Beispiel das Prinzip der fairen Chancengleichheit - nicht zeitnah genug weiter ausgearbeitet werden können, um in konkreten Fällen zu einer Lösung zu kommen. Stattdessen sollte gemeinsam in einem fairen Verfahren entschieden werden. In Situationen vernünftiger Uneinigkeit sind nach Daniels Allokationsentscheidungen also genau dann fair, wenn sie im Rahmen eines fairen Entscheidungsverfahrens (fair process) getroffen worden sind. Die Bedingungen dieses Entscheidungsverfahrens hat er gemeinsam mit James Sabin in dem Modell der >Verantwortung für Vernünftigkeit entwickelt [3, 16-18].

\section{Verantwortung für Vernünftigkeit، (accountability for reasonableness)}

Die zentral Idee hinter dem Modell der >Verantwortung für Vernünftigkeit< ist die öffentliche Verantwortung für vernünftige Allokationsentscheidungen. Zur Umsetzung dieser Idee muss ein Entscheidungsverfahren vier Bedingungen erfüllen [6, S. 118-119]:

1. Öffentlichkeit: Sowohl direkte als auch indirekte Allokationsentscheidungen und deren Begründungen müssen öffentlich zugänglich sein.

2. Relevanz: Die Begründungen für Allokationsentscheidungen müssen eine vernünftige Erklärung dafür abgeben, wie ein Leistungsanbieter unter vernünftiger Mittelknappheit den vielfältigen Gesundheitsbedürfnissen einer umschriebenen Gemeinschaft - zum Beispiel den Mitgliedern einer Versicherung - nachkommen will.

3. Revidierbarkeit und Einspruchsverfabren: Es muss Verfahren zur Anfechtung von Allokationsentscheidungen und zur Schlichtung von Konflikten geben.

4. Umsetzung: Durch freiwillige oder öffentliche Regulierung und Aufsicht muss sichergestellt sein, dass ein Entscheidungsverfahren die obigen Bedingungen erfüllt.

Verantwortung für Vernünftigkeit< wird explizit als normativer Ansatz verstanden. Wenn hinreichend spezifizierte Gerechtigkeitsprinzipien unter vernünftigen Leuten umstritten sind, sollen Allokationsentscheidungen genau dann als fair anerkannt werden, wenn sie im Rahmen eines Entscheidungsverfahrens getroffen wurden, das die vier Bedingungen von >Verantwortung für Vernünftigkeit` erfüllt.

\section{Wie führt ,Verantwortung für Vernünftigkeitı zu fairen Allokationsentscheidungen?}

Daniels beschreibt >Verantwortung für Vernünftigkeit « als einen »klassischen Appell an prozedurale Gerechtigkeit « [6, S. 109]. Allerdings gibt es unterschiedliche Formen prozeduraler Gerechtigkeit. Jede davon ist durch ein spezifisches Ver- hältnis zwischen Verteilungsverfahren und inhaltlich bestimmten Kriterien der Verteilungsgerechtigkeit definiert [15, S. 83-90]. Welche Form prozeduraler Gerechtigkeit liegt also Daniels' ,Verantwortung für Vernünftigkeit‘ zugrunde?

\subsection{Reine prozedurale Gerechtigkeit}

Modelle rein prozeduraler Gerechtigkeit haben zwei wesentliche Merkmale. Erstens gibt es kein unabhängiges Gerechtigkeitskriterium für Entscheidungen, das außerhalb eines Verfahrens zum Treffen dieser Entscheidungen ausgewiesen werden könnte (zum Beispiel durch theoretische Einsicht). Zweitens existiert ein Entscheidungsverfahren dahingehend, dass jede im Rahmen dieses Verfahrens getroffene Entscheidung gemeinhin als gerecht anerkannt wird. Zum Beispiel wird beim Roulette die Verteilung des Gewinnes genau dann als gerecht angesehen, wenn sie durch die Anzeige auf der Cuvette bestimmt wurde und der Croupier den Coup nicht manipuliert hat. Das Spiel bestimmt den gerechten Ausgang unabhängig von möglichen anderen Überlegungen, beispielsweise dass jeder einen fairen Anteil seines Einsatzes als Gewinn zurückerhalten sollte.

V Verantwortung für Vernünftigkeit ist kein Modell rein prozeduraler Gerechtigkeit, da die vier aufgestellten Bedingungen für einen fairen Entscheidungsprozess nicht rein prozedural sind. ,Verantwortung für Vernünftigkeit beinhaltet eine inhaltlich bestimmte Bedingung - die Relevanz-Bedingung

Das Modell der prozeduralen Gerechtigkeit ergänzt das Prinzip der fairen Chancengleichheit.

- die gewisse Gründe für Allokationsentscheidungen ausschließt. Darüber hinaus hebt Daniels selbst hervor, dass seine Wendung zur prozeduralen $\mathrm{Ge}$ rechtigkeit das Prinzip der fairen Chancengleichheit ergänzt, nicht aber ersetzt [6, S. 6, 27, 110, 251]. Auch führt er die Idee eines fairen Verfahrens für Allokationsentscheidungen als eine rein praktische Notwendigkeit ein. Daniels ist kein Skeptiker. Er schließt nie aus, dass 
philosophische Untersuchungen weithin anerkannte und hinreichend spezifische Prinzipien ergeben können, welche die Bedeutung von fairer Chancengleichheit im Gesundheitsbereich bestimmen [6, S. 25]. Ein faires Entscheidungsverfahren ist vor allem deswegen notwendig, weil Philosophen den Bedarf an inhaltlich bestimmten und spezifischen $\mathrm{Ge}$ rechtigkeitsprinzipien nicht so zeitnah decken können, dass Entscheidungsträger heute begründet entscheiden könnten [6, S. 108]. Dies alles spricht nicht dafür, dass >Verantwortung für Vernünftigkeit ein Modell rein prozeduraler Gerechtigkeit ist, und lenkt den Blick auf andere Modelle prozeduraler Gerechtigkeit.

\subsection{Vollkommene und unvollkommene prozedurale Gerechtigkeit}

Modelle vollkommener und unvollkommener prozeduraler Gerechtigkeit haben zwei zentrale Eigenschaften. Erstens existiert ein vom Entscheidungsverfahren unabhängiges Gerechtigkeitskriterium, mittels dessen konkrete Entscheidungen klar als gerecht oder ungerecht ausgewiesen werden können - beispielsweise das Prinzip der fairen Chancengleichheit im Kontext von Daniels' Theorie der Gesundheitsgerechtigkeit. Zweitens gibt es ein durchführbares Entscheidungsverfahren, durch das die Gerechtigkeit bzw. Ungerechtigkeit einer Entscheidung gemäß dieses unabhängigen Kriteriums ermittelt werden kann.

Im Fall vollkommener prozeduraler Gerechtigkeit kann anhand eines Entscheidungsverfahrens immer ermittelt werden, welche Entscheidung unabhängig von diesem Verfahren gerecht ist. Wenn es beispielsweise einen köstlichen Kuchen gibt, sollte dieser gleich geteilt werden - zumindest in der Regel. Die gleiche Teilung des Kuchens kann durch ein Verfahren erreicht werden, nach dem das kleinste Stück an diejenige Person geht, die den Kuchen schneidet (sofern diese Person ein möglichst großes Kuchenstück bekommen möchte, in der Lage ist, den Kuchen gleich zu schneiden, usw.). Dieser Verfahrensausgang erfüllt also das vom Verteilungsverfahren unabhängige Gerechtigkeitskriterium.

Dem gegenüber kann im Fall unvollkommener prozeduraler Gerechtigkeit in den meisten, aber nicht in allen Fällen durch ein Entscheidungsverfahren ermittelt werden, welche Entscheidung gemäß eines unabhängigen Gerechtigkeitskriteriums gerecht bzw. ungerecht ist. Die meisten Menschen sind zum Beispiel der festen Überzeugung, dass nur schuldige Personen in einem Gerichtsverfahren verurteilt werden sollten. Aber obwohl damit ein klares und unabhängiges Gerechtigkeitskritierium besteht, gibt es kein unfehlbares Verfahren, anhand dessen in jedem einzelnen Fall ermittelt werden kann, wer schuldig ist und wer nicht. Traurigerweise führen Gerichtsentscheide manchmal zur Verurteilung einer unschuldigen Person.

Daniels' , Verantwortung für Vernünftigkeit ist weder ein Modell vollkommener noch ein Modell unvollkommener prozeduraler Gerechtigkeit.

Um als ein Modell vollkommener oder unvollkommener prozeduraler Gerechtigkeit zu fungieren, müsste $>$ Verantwortung für Vernünftigkeit< ein unabhängig bestimmtes, inhaltliches Fairness-Kriterium aufweisen. Der folgende Abschnitt zeigt jedoch auf, dass die einzige inhaltlich bestimmte Bedingung von 'Verantwortung für Vernünftigkeit - die Relevanz-Bedingung - zu unklar gefasst ist, um ein solches Kriterium abzugeben. Damit ist ,Verantwortung für Vernünftigkeit` weder ein Modell vollkommener noch ein Modell unvollkommener prozeduraler Gerechtigkeit.

\subsubsection{Was bedeutet die Relevanz-Bedingung?}

Die Relevanz-Bedingung schränkt die Art von Gründen ein, die Allokationsentscheidungen in einem fairen Entscheidungsverfahren berechtigterweise zugrunde gelegt werden können [2, S. 123-31]. Die Relevanz-Bedingung schreibt vor, dass Allokationsentscheidungen nur auf der Basis von Gründen getroffen werden sollen, die von fair gesinnten (fair-minded) Leuten anerkannt werden. In seinem jüngsten Buch definiert Daniels relevante Gründe an drei Stellen [6]. Die drei Definitionen unterscheiden sich geringfügig, aber laufen letztlich alle ins Leere. Die erste Definition ist offensichtlich zirkulär. Gründe sind genau dann relevant, wenn fair gesinnte Leute übereinstimmen, dass sie für Allokationsentscheidungen relevant sind - wobei fair gesinnte Leute bei diesen Entscheidungen nur relevante Gründen anerkennen [6, S. 117-18].

Die zweite Definition erweist sich genau in denjenigen Situationen als wenig hilfreich, die einen prozeduralen Ansatz überhaupt erst notwendig machen, um konkrete Allokationsentscheidungen treffen zu können. Relevante Gründe werden von fair gesinnten Leuten anerkannt, die "sich bemühen, Wege der Kooperation unter Bedingungen zu finden,

die für alle Beteiligten tragbar sind «

[6, S. 124]. Nach Rawls ist aber die Bereitschaft, faire Kooperationsbedingungen vorzuschlagen und diesen zu folgen - sofern andere das ebenfalls tun - ein grundlegender Aspekt von Vernünftigkeit [19]. Und da wir -Verantwortung für Vernünftigkeit brauchen, weil vernünftige Leute sich darüber uneinig sind, wie notwendige Allokationsentscheidungen zu treffen sind, hilft diese zweite Definition relevanter Gründe ebenfalls wenig weiter.

Die dritte Definition besagt, dass sich relevante Gründe auf die Frage beziehen müssen, wie unter Bedingungen der Mittelknappheit Gesundheitsbedürfnissen auf eine faire Weise nachgekommen werden kann [6, S. 118]. Die sfaire Deckung von Gesundheitsbedürfnissen kann grundsätzlich so viele Bedeutungen haben wie es Konzeptionen von Fairness gibt. Ohne eine nähere Bestimmung von Fairness ist diese Definition relevanter Gründe daher offen. Allerdings ist es im Kontext von Daniels' Arbeit naheliegend, dass Fairness die Übereinstimmung mit dem Prinzip der fairen Chancengleichheit bedeutet. Allerdings kann die Übereinstimmung mit dem Prinzip fairer Chancengleichheit nicht das unabhängige und inhaltlich bestimmte Fairness-Kriterium darstellen, das für ein Modell vollkommener oder unvollkommener prozeduraler Gerechtigkeit notwendig wäre. Wenn das Prinzip fairer Chancengleichheit zu unbestimmt ist, um konkrete Allokationsentscheidungen treffen zu können, ist jede Definition, die sich auf dieses Prinzip bezieht, genauso unbestimmt. Damit ist die dritte Definition relevanter Gründe entweder offen, oder sie ist 
genauso unbestimmt wie das Prinzip der fairen Chancengleichheit.

\subsection{Eingeschränkt-reine prozedurale Gerechtigkeit}

Wenn ein Kuchen geschnitten oder in einem Verbrechen ermittelt wird, gibt es einen und nur einen einzigen gerechten Ausgang, den ein Verfahren ergeben sollte: der Kuchen soll gleich geteilt werden, und der Schuldige soll verurteilt bzw. die Unschuldige freigesprochen werden. Die Modelle vollkommener und unvollkommener prozeduraler Gerechtigkeit unterscheiden sich damit einzig darin, wie genau das gegebene Verfahren ermittelt, was in der jeweiligen Situation die gerechte Entscheidung ist. Im Gegensatz dazu ist die RelevanzBedingung der >Verantwortung für Vernünftigkeit mit mehr als nur einem einzigen fairen Ausgang vereinbar. Denn wie der letzte Abschnitt ergeben hat, ist die Relevanz-Bedingung bestenfalls so unbestimmt wie das Prinzip der fairen Chancengleichheit. Damit fehlt der >Verantwortung für Vernünftigkeit` das klare, unabhängige und inhaltlich bestimmte Fairness-Kriterium, das Modelle vollkommener oder unvollkommener prozeduraler Gerechtigkeit benötigen.

Dennoch ist das Prinzip der fairen Chancengleichheit und damit auch die Relevanzbedingung entscheidungs- und handlungsleitend. Chancengleichheit schließt bestimmte Allokationsentscheidungen aus: zum Beispiel solche, denen unberechtigte Diskriminierung aufgrund von Geschlecht oder »Rasse « zugrunde liegt. Allerdings ist Chancengleichheit vereinbar mit zahlreichen verschiedenen und gleichermaßen gerechten Handlungsmöglichkeiten. Wie der Bioethiker Ezekiel Emanuel festgehalten hat, stellen Rawls' - und damit auch Daniels' - Gerechtigkeitsprinzipien einen »Schwellentest für Gerechtigkeit " (threshold test of justice) dar [20]. Sie schränken die Spannbreite an Handlungsmöglichkeiten ein, aber sie erfordern nicht eine und nur eine einzige gerechte Entscheidung oder Handlung. Rawls selbst hebt hervor, dass die Wahl zwischen gleichermaßen gerechten Möglichkeiten nicht eine Frage der Gerechtigkeit, sondern eine Frage von fairen Entscheidungsverfahren ist [15, S. 201].

Daniels hält in seinem jüngsten Buch wiederholt am Prinzip der fairen Chancengleichheit fest [6, S. 6, 27, 110, 251]. Aus diesem Grund scheint es am plausibelsten, relevanten Gründe als solche Gründe zu verstehen, die mit den Forderungen des Prinzips der Chancengleichheit vereinbar sind. Damit würde die Relevanz-Bedingung einem »Schwellentest für Gerechtigkeit « gleichkommen, mittels dessen geprüft werden kann, ob die erwogenen Allokationsentscheidungen mit dem Prinzip der fairen Chancengleichheit vereinbar sind. Dies unterscheidet >Verantwortung für Vernünftigkeit ' grundlegend vom Roulettespiel, vom Kuchenschneiden und von der Ermittlung eines Verbrechens. Denn die im Rahmen von ,Verantwortung für Vernünftigkeit` getroffenen Entscheidungen sind weder per Definition fair, noch kann anhand von >Verantwortung für Vernünftigkeit mehr oder weniger genau ermittelt werden, was unabhängig von diesem Entscheidungsverfahren inhaltlich gerecht oder fair ist. Daniels prozeduraler Ansatz zur Lösung konkreter Gerechtigkeitsprobleme ist damit kein klassisches Modell prozeduraler Gerechtigkeit [15], sondern er begründet eine eigene Form eingeschränkt-reiner prozeduraler Gerechtigkeit. Norman Daniels stimmt dieser Lesart zu [21].

Eingeschränkt-reine prozedurale $\mathrm{Ge}$ rechtigkeit hat zwei wesentliche Merkmale. Erstens existiert ein vom Entscheidungsverfahren unabhängig bestimmtes, inhaltliches Gerechtigkeitskriterium, das mit mehreren und gleichermaßen gerechten Entscheidung vereinbar ist. Im Modell der >Verantwortung für Vernünftigkeit ‘ ist dies die RelevanzBedingung. Zweitens erlaubt ein reines Entscheidungsverfahren eine faire Wahl zwischen den Handlungsmöglichkeiten, die sich als gleichermaßen gerecht erwiesen haben. Dieses Entscheidungsverfahren macht keine inhaltlichen Vorgaben, sondern nur Verfahrensvorgaben. Die Bedingungen der Öffentlichkeit, Revidierbarkeit und der Umsetzung haben im Modell der >Verantwortung für Vernünftigkeit`diese Funktion. Damit hängt die Gerechtigkeit oder Fairness von eingeschränkt-reiner prozeduraler Gerechtigkeit sowohl von der Richtigkeit des unabhängigen Gerechtigkeitskriteriums als auch von der Fairness des
Verfahrens ab.

Eine nähere Untersuchung der inhaltlichen Bestimmung der Relevanz-Bedingung kann hier nicht geleistet werden. Daniels' Position hinsichtlich des Zusammenhangs zwischen fairer Chancengleichheit und Gesundheit ist komplex und sogar von Autoren kritisiert worden, die Rawls' Theorie der Gerech-

\section{prozeduralen Jen der ,Verantwortung ernünftigkeitı sind ungsbedürftig.}

tigkeit als Fairness vertreten [22, 23]. Bescheidener soll damit im Folgenden mit einigen kritischen Anmerkungen zu den rein prozeduralen Bedingungen von , Verantwortung für Vernünftigkeit geschlossen werden.

\section{Kritische Bemerkungen zu den rein prozeduralen Bedingungen von ,Verantwortung für Vernünftigkeit،}

\subsection{Formale Fairness}

Ein klar definiertes Entscheidungsverfahren kann die konsistente Behandlung von ähnlichen Fällen und damit formale Fairness befördern. Indem alle Allokationsentscheidungen im Rahmen eines im voraus festgelegten Entscheidungsverfahrens getroffen werden, erhöht sich die Wahrscheinlichkeit, dass in relevanter Hinsicht ähnliche Fälle tatsächlich ähnlich gehandhabt werden. Daniels selbst hebt hervor, dass ।Verantwortung für Vernünftigkeit ‘ durch die Öffentlichkeits-Bedingung formale Fairness befördert [6, S. 122]. Diese Funktion wird durch die Bedingung der Revidierbarkeit verstärkt. Wenn Allokationsentscheidungen und deren Begründungen öffentlich zugänglich gemacht werden müssen und gegen diese Entscheidungen Einspruch erhoben werden kann, dann werden Entscheidungsträger ein Interesse daran haben, ähnliche Fälle ähnlich zu behandeln bzw. die unterschiedliche Handhabung von ähnlichen Fällen stichhaltig zu begründen. Zu formaler Fairness trägt darüber hinaus die Umsetzungs-Bedingung bei, indem sie verhindert, dass die Bedingungen der Öffentlichkeit, Relevanz und Revidier- 
barkeit nicht einfach zum Vorteil von Entscheidungsträgern geändert oder aufgegeben werden können.

Dennoch sind die Bedingungen von Verantwortung für Vernünftigkeit meines Erachtens nicht hinreichend, um formale Fairness von Allokationsentscheidungen zu gewährleisten. Denn mit dem Prinzip der fairen Chancengleichheit können widersprüchliche Entscheidungen vereinbar sein - wie zum Beispiel Entscheidungen für oder gegen eine Rückerstattung der Kosten für die neue Chemotherapie. Daniels stellt richtigerweise fest, dass Versicherungsträger oder Leistungserbringer im Gesundheitswesen bei Mittelknappheit $\mathrm{zu}$ verschiedenen (und zuweilen widersprüchlichen Entscheidungen) kommen können, und dass diese Entscheidungen genau dann nicht gegen formale Fairness verstossen, wenn das zugrunde gelegte Entscheidungsverfahren fair war. Dies gilt sogar dann, wenn die Fälle in relevanter Hinsicht ähnlich sind [6, S. 135-37]. Aber was, wenn dieselbe Institution in ähnlichen Fällen unterschiedliche Allokationsentscheide trifft? Angenommen die GKV würde in der ersten Jahreshälfte die Kosten für die neue Chemotherapie rückerstatten (beispielsweise basierend auf dem Argument der letzten Chance), aber in der zweiten Jahreshälfte Kosteneffektivitätsüberlegungen den Vorrang geben und die Kosten nicht rückerstatten. Beide Allokationsentscheidungen sind mit dem Prinzip der fairen Chancengleichheit vereinbar, aber sie verstoßen gegen die Anforderungen formaler Fairness. Die Bedingungen der Öffentlichkeit, Revidierbarkeit und Umsetzung können solche Szenarien wahrscheinlich nicht vermeiden - vor allem dann nicht, wenn die Öffentlichkeits-Bedingung keine aktive Kommunikation von Allokationsentscheidungen erfordert. Eine Konsistenz-Bedingung sollte daher von jeder Institution im Gesundheitsbereich konsistente oder wenigstens bestmöglich konsistente Allokationsentscheidungen fordern.

\subsection{Einschluss repräsentativer Stellvertreter}

Wenn Allokationsentscheidungen zwischen gleichermaßen gerechten Möglichkeiten Fragen eines fairen Verfahrens sind - und nicht Fragen der Gerechtig- keit - dann ist der Einschluss repräsentativer Stellvertreter in die entsprechenden Entscheidungsverfahren entscheidend. Allerdings müssten die rein prozeduralen Bedingungen von >Verantwortung für Vernünftigkeit` meines Erachtens aus mehreren Gründen weiter spezifiziert und ergänzt werden, damit die Gesundheitsbedürfnisse aller Mitglieder einer Gemeinschaft im Entscheidungsverfahren fair berücksichtigt werden.

Erstens fordert die ÖffentlichkeitsBedingung nur, dass Allokationsentscheidungen und deren Begründungen öffentlich zugänglich gemacht werden müssen. Dies setzt voraus, dass die Öffentlichkeit oder die Mitglieder definierter Gemeinschaften - wie zum Beispiel die Mitglieder einer bestimmten Krankenversicherung - bereits wissen, dass Allokationsentscheidungen getroffen werden. Aber öffentliches Bewusstsein über diese Entscheidungen existiert möglicherweise nicht immer. Und wenn es existiert, sind viele Menschen einfach zu passiv, um die entsprechenden Informationen zu suchen oder einzufordern. Entgegen Daniels' Annahmen ist aktive Kommunikation von Allokationsentscheidungen notwendig (zum Beispiel durch Rundbriefe, Internet-basierte Diskussionsforen oder öffentliche Veranstaltungen), um die ÖffentlichkeitsBedingung zu erfüllen. Effektive Öffentlichkeit ist darüber hinaus auch eine Voraussetzung für die RevidierbarkeitsBedingung, denn um Einspruch erheben zu können, muss man von Allokationsentscheidungen wissen.

Zweitens sind die Bedingungen von ,Verantwortung für Vernünftigkeit nicht hinreichend, um einen angemessenen Einschluss repräsentativer Stellvertreter in den Verteilungsprozess zu gewährleisten. >Verantwortung für Vernünftigkeit< sieht vor, dass Gesundheitsinstitutionen Allokationsentscheidungen treffen und hierfür ihre Gründe angeben. Dem gegenüber können diejenigen, die von diesen Entscheidungen betroffen sind, nur Einspruch erheben. Daniels beharrt darauf, dass die öffentliche Beteiligung am Entscheidungsverfahren allein dazu dient, den Abwägungs- und Entscheidungsprozess inhaltlich zu bereichern und einmal getroffene Alloka-

tionsentscheidungen $\mathrm{zu}$ verbreiten [6, S. 129-31]. Damit hat die öffentliche Beteiligung für Daniels rein instrumentellen Wert. Öffentliche Beteiligung ist an sich nicht wertvoll, so Daniels, weil demokratisch-repräsentative Strukturen oder Verfahren zur Auswahl von Stellvertretern "völlig fehlen« [6, S. 129].

Diese Argumentation ist allerdings nicht überzeugend. Denn obwohl bestehende Verfahren zur Repräsentation der Öffentlichkeit sicher weit davon entfernt sind, ideal zu sein, fehlen sie in demokratischen Gesellschaften nicht völlig. Darüber hinaus schließt das Fehlen eines absolut vollkommenen Verfahrens nicht den Gebrauch eines durchführbaren unvollkommenen Verfahrens aus. Zum Beispiel schaffen wir das Strafrecht nicht deswegen ab, weil es manchmal (und traurigerweise) zur Bestrafung einer unschuldigen Person führt. Insofern als bestehende Verfahren zur Beteiligung repräsentativer Stellvertreter begrenzt sind, ist dies vielmehr ein Grund kreativer zu werden. Beteiligung in Debatten über Finanzierungsschwerpunkte im Gesundheitsbereich, Kommunikation mit politischen Vertretern, Bildung von Interessensverbänden zum Lobbying und Fürsprechen, usw. - all dies sind Wege, Entscheidungsverfahren zu beeinflussen und die faire Berücksichtigung der Gesundheitsbedürfnisse aller Gemeinschaftsmitglieder zu befördern [24]. Eine Bedingung der öffentlichen Beteiligung sollte daher fordern, dass solche Einflussmechanismen entwickelt und eingesetzt werden.

Drittens müsste das Abstimmungsverfahren weiter spezifiziert werden. Die Relevanz-Bedingung sieht vor, dass

\section{Das Modell iVerantwortung für Vernünftigkeits ist der wahrscheinlich einflussreichste Ansatz für faire Entscheidungsverfahren im Gesundheitswesen.}

in Fällen unlösbarer vernünftiger Uneinigkeit über Allokationsentscheidungen abgestimmt werden kann. Allerdings macht Daniels keine genaueren Angaben zum Abstimmungsverfahren, sondern deutet nur an, dass bei Abstimmun- 
gen eine »einfache (oder vielleicht eine absolute oder qualifizierte) Mehrheit « entscheidend sein soll [6, S. 113]. Diese Vagheit könnte aber nicht nur relevante Unterschiede zwischen Entscheidungssituationen übergehen, die verschiedene Abstimmungsverfahren erfordern würden (wie zum Beispiel die Anzahl der Wahlmöglichkeiten). Sie könnte in der Praxis auch zu willkürlichen Abstimmungsverfahren und damit zu willkürlichen Allokationsentscheidungen führen. Da alle Handlungsmöglichkeiten ohnehin gleichermaßen gerecht sind, könnte man meinen, dass dies unproblematisch sei. Aber ohne klar definierte Abstimmungsverfahren sind konsistente Entscheidungen nicht möglich. Konkretere Handlungsanweisungen sind hier notwendig.

Viertens sollten diejenigen, die Allokationsentscheidungen ausarbeiten und in die Praxis umsetzen, nicht derart von ihren eigenen Interessen getrieben sein, dass sie konkurrierende Erwägungen nicht mehr abwägend gewichten können [24]. Obwohl die Relevanz-Bedingung Verstöße gegen das Prinzip der fairen Chancengleichheit ausschließen soll, ist es immer noch denkbar, dass größere Interessenkonflikte Entscheidungen zwischen gleichermaßen gerechten Optionen übergebührlich beeinflussen. Eine relative Unparteilichkeits-Bedingung sollte diese Möglichkeit ausschließen.

\section{Schlussfolgerung}

Norman Daniels' und James Sabin's Modell der >Verantwortung für Vernünftigkeit ist der wahrscheinlich einflussreichste Ansatz für faire Entscheidungsverfahren im Gesundheitswesen. Doch obwohl der Ansatz in der Prioritätensetzung oft verwendet wird und auch aus theoretischer Perspektive breit diskutiert ist, hat bis anhin vor allem die Relevanz-Bedingung viele Fragen aufgeworfen. Dieser Beitrag hat die Relevanz-Bedingung einer kritischen Analyse unterzogen und ihre beschränkende, aber nicht klar als gerecht bzw. ungerecht ausweisende Funktion in Allokationsentscheidungen herausgearbeitet. Aus dieser - auch von Norman Daniels geteilten Lesart - ergibt sich, dass die >Verantwortung für Vernünftigkeit eine neue Form der eingeschränkt-reinen prozeduralen Gerechtigkeit begründet. Der Beitrag hat darüber hinaus argu- mentiert, dass die rein prozeduralen Bedingungen von >Verantwortlichkeit für Vernünftigkeit< weiter spezifiziert und erweitert werden müssen, um insgesamt ein faires Entscheidungsverfahren zu ermöglichen. In diesem Zusammenhang wurden einige konkrete und praktische relevante Erweiterungen vorgestellt (Konsistenz-Bedingung, Bedingung der öffentlichen Beteiligung, Unparteilichkeits-Bedingung). Damit ist der Ansatz der $>$ Verantwortlichkeit für Vernünftigkeit< sowohl theoretisch als auch praktisch besser greifbar geworden. Es wäre wünschenswert, wenn die Überlegungen in diesem Beitrag die Diskussion um faire Entscheidungsprozesse auch in Deutschland vorantreibt.

\section{Literatur}

1. Rid, A., Justice and Procedure: How Does "Accountability for Reasonableness « result in fair limit-setting decisions? Journal of Medical Ethics, 2009. 35(1): 12-16.

2. Rid, A., Faire Allokationsprozesse: zum Verhältnis von substantieller und prozeduraler Gerechtigkeit in Norman Daniels' Just Health. In: Strech, D. und Marckmann, G. (Hrsg.): Public Health Ethik. 2010, Münster: LIT Verlag. S. 159-171.

3. Daniels, N. and J. Sabin, Limits to Health Care: Fair Procedures, Democratic Deliberation, and the Legitimacy Problem for Insurers. Philosophy and Public Affairs, 1997. 26(4): 303-350.

4. World Health Organization, Joint United Nations Programme on HIVIAIDS, Guidance on ethics and equitable access to HIV treatment and care. 2004, Geneva: World Health Organization. Erhältlich unter: http://www.who.int/ethics/publications/en/ (abgerufen am 18.4.2012).

5. National Institute for Health and Clinical Excellence, Social Value Judgments: Principles for the Development of NICE Guidance (Second Edition). 2008, London: National Institute for Health and Clinical Excellence. Erhältlich unter: http://www. nice.org.uk/aboutnice/howwework/ socialvaluejudgements/socialvaluejudgements.jsp (abgerufen am 18.4.2012).

6. Daniels, N., Just Health: Meeting Health Needs Fairly. 2008, New York: Cambridge University Press.

7. de Bont, A., Zandewijken, G., Stolk, E., and Niessen, L., Prioritisation by physicians in the Netherlands - the growth hormone example in drug reimbursement decisions. Health Policy, 2007. 80 369-377.

8. Gibson, J. L., Martin, D. K. and Singer, P. A., Priority setting in hospitals: fairness, inclusiveness, and the problem of institu tional power differences. Social Science \& Medicine, 2005. 61(11): 2355-2362.

9. Kapiriri, L., and Norheim, O. F., Criteria for priority-setting in health care in Uganda: exploration of stakeholders' values. Bulletin of the World Health Organization, 2003. 82(2): 172-179.

10. Martin, D. K., Giacomini, M. and Singer, P. A., Fairness, accountability for reasonableness, and the views of priority setting decision-makers. Health Policy, 2002. 61 279-290.
11. Kapiriri, L., Norheim O.F., and Martin, D. K., Fairness and accountability for reasonableness. Do the views of prioritysetting decision makers differ across health systems and levels of decision making? Social Science \& Medicine, 2009 68: 766-773.

12. Hasman, A. and S. Holm, Accountability for Reasonableness: Opening the Black Box of Process. Health Care Analysis, 2005. 13(4): 261-273.

13. Friedman, A., Beyond Accountability for Reasonableness. Bioethics, 2008. 22(2): 101-12.

14. Lauridsen, S., Lippert-Rasmussen, K., Legitimate Allocation of Public Healthcare: Beyond Accountability of Reasonableness. Public Health Ethics, 2009. 2(1): 59-69.

15. Rawls, J., A Theory of Justice. 1971, Cambridge, MA, London: Harvard University Press

16. Daniels, N. and J. Sabin, The Ethics of Accountability in Managed Care Reform. Health Affairs, 1998. 17(5): 50-64.

17. Daniels, N. and J.E. Sabin, Last Chance Therapies and Managed Care: Pluralism, Fair Procedures, and Legitimacy. Hastings Cent Rep, 1998. 28(2): 27-41.

18. Daniels, N. and J.E. Sabin, Setting Limits Fairly. Can We Learn to Share Medical Resources? 2 ed. 2007, New York: Oxford University Press.

19. Rawls, J., Political Liberalism (expanded edition). 2005, New York: Columbia University Press: 48-54

20. Emanuel, E.J., The Ends of Human Life. 1991, Cambridge, London: Harvard University Press: 146.

21. Daniels, N., Just Health: Replies and Further Thoughts. Journal of Medical Ethics, 2009. 35(1): 36-41.

22. DeGrazia, D., Grounding a Right to Health Care in Self-Respect and Self-Esteem. Public Affairs Quarterly, 1991. 5(4): 301-318.

23. Sachs, B., The Liberty Principle and Universal Health Care. Kennedy Institute of Ethics Journal, 2008. 18(2): 149-72.

24. Emanuel, E.J., Book review of Setting Limits Fairly: Can We Learn to Share Medical Resources? By Norman Daniels and James E. Sabin. New England Journal of Medicine, 2002. 347(12): 952-954. 